\title{
Transitioning from gavage to full oral feeds in premature infants: When should we discontinue the nasogastric tube?
}

\author{
Sreekanth Viswanathan $\mathbb{1}^{1,2} \cdot$ Sudarshan Jadcherla ${ }^{1,2}$
}

Received: 16 May 2019 / Revised: 9 June 2019 / Accepted: 12 June 2019 / Published online: 31 July 2019

(c) The Author(s), under exclusive licence to Springer Nature America, Inc. 2019

\begin{abstract}
Background The optimal timing for discontinuation of nasogastric (NG) tube in premature infants transitioning to oral feeding is not known.

Objective To determine whether early removal of NG-tube is appropriate in low-risk premature infants.

Methods Prospectively collected data of premature infants started on oral feeds at $\leq 34$ weeks gestation were reviewed. Infants were categorized into 'early' or 'late' NG-removal groups based on the proportion of oral intake in the preceding 2-days, i.e., $60-79 \%$ or $80-100 \%$ of the total volume, respectively.

Results In total 50 infants in early group vs. 43 in late group. Both groups had similar oral intake and weight change in the subsequent 2-days post-NG removal. The days from NG-removal to target oral volume, and to hospital discharge trended shorter in early vs. late group.

Conclusions Discontinuing NG-tube when the oral feeding competency reaches $\sim 75 \%$ of prescribed feeding volume is safe and appropriate in low-risk premature infants.
\end{abstract}

\section{Introduction}

Successful transition from full gavage to independent oral feedings is a criterion of hospital discharge in premature infants [1]. Delay in achieving full oral feeding milestone is one of the main reasons for delays in hospital discharge [24], and adverse neurodevelopmental outcome in premature infants [5]. During this period of functional immaturity of coordinating sucking, swallowing, and breathing [6], many premature infants require enteral feeding, usually by a nasogastric (NG) tube, until they achieve adequate oral feeding skills. The optimal timing for discontinuation of NG tube feeding in premature infants transitioning to oral feeding is not known, and thus associated with practice variation.

Sreekanth Viswanathan

sreepeds@gmail.com

1 Division of Neonatology, Department of Pediatrics, Nationwide Children's Hospital, The Ohio State University College of Medicine, Columbus, OH, USA

2 Neonatal and Infant Feeding Disorders Research Program, Center for Perinatal Research, The Research Institute at Nationwide Children's Hospital, Columbus, OH, USA
Some of the cue-based oral feeding protocols suggest to keep the NG feeding tube until the infant have appropriate weight gain for 48 hours without supplementation by NG tube [7-9]. However, having a NG tube is not without problems. In presence of NG tube especially with size $>5 \mathrm{~F}$, premature infants have increased nasal airway resistance $[10,11]$, and reduced tidal volume and minute ventilation during sucking, which can affect their oral feeding performance [12]. Its presence can potentially increase the gastroesophageal reflux (GER) events depending on the size of NG tube $[13,14]$. Use of NG tube is also associated with significant nutrient loss, especially fat, fat soluble vitamins, and minerals because fat adheres to tubing surfaces [15], and is a potential source of infection as it is easily colonized by pathogenic bacteria/fungi [16].

If earlier removal of NG tube is safe and feasible, it can potentially reduce these complications and length of hospital stay in premature infants. Our study objective was to determine whether early removal of NG tube is safe and appropriate in relatively low-risk premature infants transitioning to oral feeding. We hypothesize that earlier removal of NG tube would lead to earlier transition to independent oral feeding resulting in decreased length of hospital stay. 


\section{Methods}

The study was conducted at the all-referral level IV neonatal intensive care unit (NICU) at Nationwide Children's Hospital, Columbus, Ohio. As part of an ongoing quality improvement (SIMPLE feeding initiatives) [17], focused on prevention and early identification of feeding related problems in premature infants, data of all infants admitted at $\leq 34$ weeks' post-menstrual age (PMA) were collected prospectively. NG tube sizes $6.5 \mathrm{~F}$ or $8.0 \mathrm{~F}$ was used for enteral feeding and feedings were typically given intermittently over 10-30min every $3 \mathrm{~h}$. There was no unit guideline regarding the timing of NG tube removal during the study period from Jan 2011 to Dec 2017. In general, the decision to remove NG tube occurs when the oral feeding volume reaches $60-100 \%$ of prescribed enteral feeding depending on the individual provider comfort level.

To meet the objective of this study, SIMPLE feeding program database was reviewed for 'low-risk' premature infants which we defined as infants born $\leq 32$ weeks' gestation and had started on cue based oral feeds at $\leq 34$ weeks' PMA. Infants on invasive respiratory support ( $>2 \mathrm{~L}$ of nasal cannula support, CPAP or ventilator support), gastrointestinal surgical conditions, intraventricular hemorrhage $>$ grade 2, and major congenital anomalies were excluded. Among eligible infants, the day of NG tube removal was recorded and the percentage of oral feeding volume (OFV) in the preceding two days of NG tube removal was calculated from the electronic medical records. Infants were categorized into 'early' and 'late' NG removal groups. In early group, NG tube was removed when the mean OFV was between $60-79 \%$ of prescribed feeding volume in the preceding 2 days, while in late group, it occurred at $80-100 \%$. Infants with mean OFV less than $60 \%$ in the preceding 2 days of NG removal were excluded considering their relatively quicker progression and more mature oral feeding skills. For both groups, characteristics before and at NG removal were collected from the SIMPLE feeding program database and electronic medical records. The clinical outcomes including time from NG removal to reach the target full oral feeding volume (ability to take PO of all prescribed enteral feeding volume, which generally ranges from 130 to $150 \mathrm{ml} / \mathrm{kg}$ ) and to NICU discharge, and growth metrics were compared between the two groups. OFV in infants who are on direct breast feeding was considered as $150 \mathrm{ml} / \mathrm{kg}$ after the NG removal. The project was approved by the Nationwide Children's Hospital Institutional Review Board.

\section{Statistics}

Appropriate bivariate analysis was performed to identify the unadjusted differences between the cases and controls.
Student's $t$-test was used for parametric continuous variables, and the Mann-Whitney $\mathrm{U}$ test was used for nonparametric continuous variables. Chi-square tests and Fisher exact tests were used for categorical variables. All quantitative data were expressed as the mean \pm standard deviation (SD), or median with inter-quartile range. A $p \leq$ 0.05 was considered to be statistically significant. The statistical software IBM SPSS Statistics version 23 (SPSS, Chicago, IL) was used for the statistical analysis of the data.

\section{Results}

Of the 93 eligible infants during the study period, 50 infants were in early NG removal group, the rest 43 were in late NG removal group. Early group had similar perinatal and birth characteristics, compared to the late group except that late group were exposed to more number of CPAP days (Table 1). At NG tube removal, early group had similar corrected gestational age, and body weight compared to the late group (Table 1). Early group had similar direct breast feeding rate at NG removal compared to late group (30.0 vs. $16.3 \%, p=0.12$ ). The mean OFV $\%$ in the preceding two days of NG removal was $72.3 \pm 5.8$ in the early group vs. $87.3 \pm 4.0$ in the late group $(p<0.001)$. The oral feeding volume and weight change in the subsequent two days after NG removal were similar in both groups (Fig. 1, Table 2). Supplemental oxygen use at initial oral feeding, at NG tube removal, at 36 weeks' gestation, and at NICU discharge were similar in both groups. Majority of the infants reached full oral target volume before discharge $(90 \%$ in early vs. 88.4 in late group, $p=0.80$ ). Early group showed a trend for lower number of days from the NG removal to reach oral target volume (median days of 2.0 vs $2.5, p=0.13$, mean days $2.4 \pm 1.9$ vs. $4.4 \pm 5.8, p=$ 0.051 ), and to NICU discharge (mean days of 10.9 vs. $13.5, p=0.21$ ), compared to late group. None required replacing NG tube in both groups, and none required tube feeding at discharge. The NICU discharge outcomes including BPD (oxygen requirement at 36 week PMA), length of hospital stay (LOHS), OFV, and discharge weight were similar in both groups (Table 2). A subgroup analysis of infants born $\leq 28$ weeks and $>29$ weeks also did not show differences between early and late groups (Table 3).

\section{Discussion}

The purpose of this study was to determine whether early removal of NG tube is safe and appropriate in relatively 
Table 1 Characteristics before and at NG removal in early and late NG removal groups

\begin{tabular}{|c|c|c|c|}
\hline Characteristics & $\begin{array}{l}\text { Early group } \\
(n=50)\end{array}$ & $\begin{array}{l}\text { Late Group } \\
(n=43)\end{array}$ & $P$ valu \\
\hline \multicolumn{4}{|l|}{ Before NG tube removal } \\
\hline Birth gestation (weeks) & $28.9 \pm 2.5$ & $28.3 \pm 2.3$ & 0.24 \\
\hline Birth weight (g) & $1333.4 \pm 422.2$ & $1214.7 \pm 365.0$ & 0.16 \\
\hline$\leq 28$ weeks gestation $(\%)$ & $20(40 \%)$ & $24(55.8)$ & 0.15 \\
\hline Male gender $(\%)$ & $28(56.0)$ & 55.8 & 0.99 \\
\hline $\begin{array}{l}\text { Small for gestational } \\
\text { age }(\%)\end{array}$ & $1(2.0)$ & $1(2.3)$ & 1.00 \\
\hline $\begin{array}{l}\text { Large for gestational } \\
\text { age }(\%)\end{array}$ & $8(16.0)$ & $4(9.5)$ & 0.37 \\
\hline $\begin{array}{l}\text { Born by Caesarian } \\
\text { section }(\%)\end{array}$ & $35(70.0)$ & $29(67.4)$ & 0.79 \\
\hline Maternal diabetes $(\%)$ & $2(4.0)$ & $4(9.3)$ & 0.41 \\
\hline Intubation (\%) & $29(58.0)$ & $28(65.1)$ & 0.48 \\
\hline Intubation days* & $1(0-2)$ & $1(0-2)$ & 0.49 \\
\hline CPAP $(\%)$ & $41(82.0)$ & $34(79.1)$ & 0.72 \\
\hline CPAP days & $11.0 \pm 14.2$ & $17.5 \pm 16.5$ & 0.05 \\
\hline IVH grade 1 or $2(\%)$ & $12(24.0)$ & $11(25.6)$ & 0.86 \\
\hline PMA at initial oral feeding & $33.3 \pm 0.5$ & $33.6 \pm 0.6$ & 0.03 \\
\hline $\begin{array}{l}\text { Human milk at } \\
\text { initial PO }(\%)\end{array}$ & $36(72.0)$ & $24(55.8)$ & 0.10 \\
\hline $\begin{array}{l}\text { Oxygen use at } \\
\text { initial PO (\%) }\end{array}$ & $21(42.0)$ & $25(58.1)$ & 0.12 \\
\hline \multicolumn{4}{|l|}{ At NG tube removal } \\
\hline PMA (weeks) & $36.2 \pm 2.3$ & $36.1 \pm 1.9$ & 0.89 \\
\hline Weight $(\mathrm{g})$ & $2446.2 \pm 457.9$ & $2370.4 \pm 471.8$ & 0.44 \\
\hline $\begin{array}{l}\text { Weight }<10^{\text {th }} \\
\text { percentile }(\%)\end{array}$ & $7(14)$ & $5(11.9)$ & 1.00 \\
\hline Human milk use (\%) & $16(32.0)$ & $16(37.2)$ & 0.60 \\
\hline Direct breast feeding $(\%)$ & $15(30)$ & $7(16.3)$ & 0.12 \\
\hline Oral feeding calories/oz & $24.1 \pm 1.3$ & $24.7 \pm 1.8$ & 0.11 \\
\hline Oxygen use (\%) & $23(46.0)$ & $16(37.2)$ & 0.39 \\
\hline OFV \% 2-days prior & $68.6 \pm 13.2$ & $84.6 \pm 8.6$ & 0.00 \\
\hline OFV \% 1-day prior & $76.0 \pm 5.8$ & $90.0 \pm 8.4$ & 0.00 \\
\hline OFV \% preceding 2-days & $72.3 \pm 5.8$ & $87.3 \pm 4.0$ & 0.00 \\
\hline
\end{tabular}

Values stated as mean \pm SD or as stated otherwise. "Median with interquartile range

$N G$ nasogastric, $I V H$ Intraventricular hemorrhage, $P M A$ Postmenstrual age, $P O$ Per Oral, $O F V$ Oral Feeding Volume

low-risk premature infants transitioning to oral feeding. By using a prospectively collected clinical database of premature infants enrolled in the SIMPLE feeding program at our all-referral NICU, our study suggests that discontinuing NG tube when the oral feeding competency reaches $\sim 75 \%$ of prescribed enteral feeding volume in the preceding two days is safe and appropriate in low-risk premature infants. The practice of keeping the NG tube until these infants reach their full targeted oral feeding
Percentage of Oral Feeding Volume (OFV) in Early vs. Late Nasogastic Tube Removal Groups

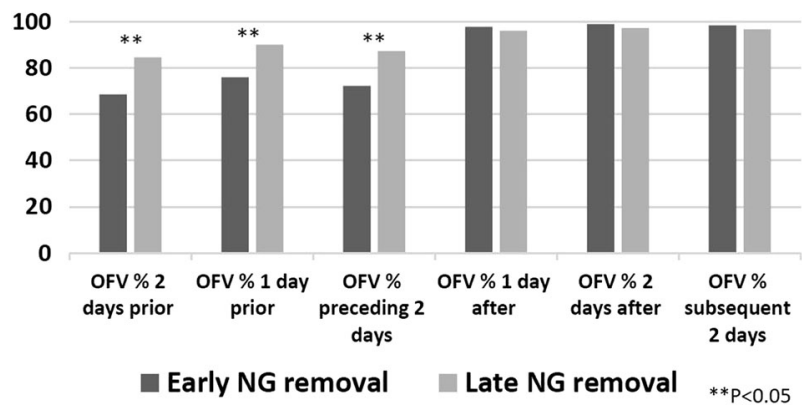

Fig. 1 Percentage of oral feeding volume (OFV) in early vs. late nasogastic tube removal groups

volume is not associated with any significant clinical benefits.

In routine clinical practice, the decision to remove the NG tube is often based on the oral feeding performance of infants in the preceding days. The relationship between chronological age and functional maturity of the oral feeding skills is no $t$ always linear, and is often influenced by growth metrics and associated co-morbidities [18]. Because of the positive association between postnatal growth and neurodevelopmental outcomes [19], potential for faltering growth with early NG tube removal is often a concern among neonatal providers. However, our data of relatively stable low-risk premature infants who have made steady oral feeding progression suggest that discontinuing NG tube when the OFV reaches about $3 / 4$ of the prescribed enteral feeding volume was not associated with significant reduction in time to achieve targeted oral feeding volume or subsequent postnatal growth until NICU discharge. Even though the late group infants were exposed to more number of CPAP days suggesting more severe early lung disease, the use of supplemental oxygen need during the oral feeding period, at 36 weeks, and at hospital discharge were similar between the two groups. Similar to our study, Fucile et al. [20], reported that setbacks in oral feeding progression are least likely to occur when the oral feeding attempt frequency reaches 6-8/day vs. 1-2 or 3-5 per day. In our center, we generally consider hospital discharge, once infant is $48 \mathrm{~h}$ off-NG tube feeding if there are no other medical or social reasons. However, infants in both our study groups were discharged from the NICU about 11-13 days after the NG tube removal. This suggests that attaining oral feeding competency is not the only reason that was delaying NICU discharge in our study population. Considering our NICU being an all-referral center, the complexity of referred infants' initial NICU course, incidence of clinical BPD at 36 weeks PMA in about $1 / 3$ of study infants, and other unmeasured medical and social variables may have 
Table 2 Outcomes after NG tube removal and at NICU discharge in early and late NG removal groups

\begin{tabular}{llll}
\hline Outcomes & Early Group $(n=50)$ & Late Group $(n=43)$ & $P$ value \\
\hline After NG tube removal & & & 0.20 \\
OFV \% 1-day after & $97.8 \pm 7.2$ & $96.1 \pm 5.4$ & 0.35 \\
OFV \% 2-days after & $99.0 \pm 9.6$ & $97.3 \pm 7.7$ & 0.22 \\
OFV \% subsequent 2-days & $98.4 \pm 7.1$ & $96.7 \pm 5.9$ & 0.78 \\
$\Delta$ Weight 1-day after (g) & $28.2 \pm 34.2$ & $30.4 \pm 40.1$ & 0.31 \\
$\Delta$ Weight in 2-days after (g) & $56.3 \pm 55.8$ & $67.1 \pm 44.0$ & 0.39 \\
Target full OFV (ml/kg) & $144.0 \pm 7.8$ & $145.3 \pm 7.3$ & 0.80 \\
Reached target OFV (\%) & $45(90.0)$ & $38(88.4)$ & 0.13 \\
Days to reach target OFV & $2.0(1-3)$ & $2.5(1-5)$ & 0.80 \\
Days from initial PO to NG removal & $19.8 \pm 14.5$ & $19.0 \pm 13.6$ & \\
At NICU Discharge & & & 0.16 \\
BPD (at 36 weeks PMA, \%) & $15(30.0)$ & $19(44.2)$ & 0.49 \\
OFV at discharge & $151.7 \pm 14.7$ & $154.1 \pm 19.3$ & 0.21 \\
Days from NG removal to discharge & $10.9 \pm 7.1$ & $13.5 \pm 11.6$ & 0.86 \\
Length of NICU stay (days) & $64.4 \pm 28.6$ & $63.2 \pm 34.9$ & 0.82 \\
Discharge weight (g) & $2715.6 \pm 529.4$ & $2742.0 \pm 564.5$ & 0.58 \\
PMA at discharge (weeks) & $37.7 \pm 2.7$ & $38.0 \pm 2.5$ & 0.07 \\
Discharged on oxygen (\%) & $11(22.0)$ & $17(39.5)$ &
\end{tabular}

Values stated as mean \pm SD or as stated otherwise. * Median with interquartile range

$N G$ nasogastric, $P M A$ Postmenstrual age, $P O$ Per Oral, $O F V$ Oral Feeding Volume, BPD Bronchopulmonary dysplasia

Table 3 Subgroup analysis stratified by GA (GA $\leq 28$ vs. $>29$ weeks) of characteristics in the early and late NG removal groups

\begin{tabular}{|c|c|c|c|c|c|c|}
\hline \multirow[b]{2}{*}{ Characteristics } & \multicolumn{3}{|l|}{$\leq 28$ weeks } & \multicolumn{3}{|l|}{$>29$ weeks } \\
\hline & $\begin{array}{l}\text { Early Group } \\
(n=20)\end{array}$ & $\begin{array}{l}\text { Late Group } \\
(n=24)\end{array}$ & $\mathrm{P}$ value & $\begin{array}{l}\text { Early Group } \\
(n=30)\end{array}$ & $\begin{array}{l}\text { Late Group } \\
(n=19)\end{array}$ & $P$ value \\
\hline Birth gestation (weeks) & $26.1 \pm 1.4$ & $26.6 \pm 1.6$ & 0.26 & $30.7 \pm 0.9$ & $30.4 \pm 0.9$ & 0.20 \\
\hline PMA at initial PO (weeks) & $33.5 \pm 0.4$ & $33.7 \pm 0.6$ & 0.19 & $33.2 \pm 0.5$ & $33.4 \pm 0.5$ & 0.26 \\
\hline PMA at NG removal (weeks) & $37.4 \pm 2.8$ & $36.8 \pm 2.2$ & 0.42 & $35.3 \pm 1.5$ & $35.2 \pm 0.7$ & 0.72 \\
\hline OFV \% 2-days prior & $69.9 \pm 16.5$ & $84.3 \pm 9.2$ & .002 & $67.6 \pm 10.7$ & $85.0 \pm 7.9$ & $<0.001$ \\
\hline OFV \% 1-day prior & $74.4 \pm 12.4$ & $91.2 \pm 8.7$ & $<0.001$ & $77.1 \pm 10.2$ & $88.6 \pm 7.9$ & $<0.001$ \\
\hline OFV \% preceding 2-days & $72.2 \pm 5.6$ & $87.7 \pm 4.3$ & $<0.001$ & $72.4 \pm 6.0$ & $86.8 \pm 3.7$ & $<0.001$ \\
\hline OFV \% 1-day after & $96.6 \pm 8.4$ & $95.2 \pm 5.9$ & 0.53 & $98.6 \pm 6.2$ & $97.1 \pm 4.6$ & 0.38 \\
\hline OFV \% 2-days after & $99.4 \pm 7.8$ & $96.8 \pm 8.4$ & 0.59 & $98.8 \pm 10.7$ & $98.0 \pm 7.0$ & 0.77 \\
\hline OFV \% subsequent 2 -days & $97.9 \pm 6.4$ & $96.0 \pm 6.4$ & 0.32 & $98.7 \pm 7.7$ & $97.6 \pm 5.3$ & 0.58 \\
\hline$\Delta$ Weight 1-day after $(\mathrm{g})$ & $32.3 \pm 38.3$ & $35.5 \pm 40.1$ & 0.79 & $25.5 \pm 31.5$ & $23.6 \pm 40.2$ & 0.06 \\
\hline$\Delta$ Weight in 2-days after (g) & $51.8 \pm 67.1$ & $71.1 \pm 45.3$ & 0.26 & $59.3 \pm 47.8$ & $61.7 \pm 42.9$ & 0.07 \\
\hline Target full OFV (ml/kg/day) & $142.0 \pm 8.3$ & $143.1 \pm 8.1$ & 0.62 & $145.3 \pm 7.3$ & $148.2 \pm 5.1$ & 0.12 \\
\hline Days to reach target $\mathrm{OFV}^{*}$ & $1.5(1.0-2.0)$ & $4.0(1.0-6.3)$ & 0.06 & $2.0(1.0-3.3)$ & $2.0(1.0-3.5)$ & 0.78 \\
\hline BPD (at 36 weeks PMA, \%) & $12(60.0)$ & $13(54.2)$ & 0.70 & $3(10.0)$ & 6 31.6) & 0.07 \\
\hline $\mathrm{OFV}$ at discharge $(\mathrm{ml} / \mathrm{kg} /$ day $)$ & $148.6 \pm 13.6$ & $156.9 \pm 22.3$ & 0.13 & $153.7 \pm 15.3$ & $150.5 \pm 14.3$ & 0.46 \\
\hline $\begin{array}{l}\text { Days from NG removal to } \\
\text { discharge }\end{array}$ & $14.1 \pm 8.8$ & $14.5 \pm 9.9$ & 0.89 & $8.8 \pm 4.9$ & $12.2 \pm 13.5$ & 0.31 \\
\hline PMA at discharge (weeks) & $39.5 \pm 3.0$ & $38.9 \pm 2.4$ & 0.49 & $36.6 \pm 1.6$ & $36.9 \pm 2.2$ & 0.51 \\
\hline
\end{tabular}

Values stated as mean \pm SD or as stated otherwise. * Median with interquartile range

$N G$ nasogastric, PMA Postmenstrual age, $P O$ Per Oral, $O F V$ Oral Feeding Volume, BPD Bronchopulmonary dysplasia 
affected the timing of NICU discharge. However, considering our specific inclusion and exclusion criteria and that all our study patients were started on oral feeding $\leq 34$ weeks' PMA suggest that our study cohort represents the relatively low-risk premature infants in a contemporary academic NICU hospital setting. Though the described approach and conclusions are limited to the select inclusions and exclusions of our study population, we believe, these findings may be relevant to most level-II NICUs or step-down units where the majority of such low-risk premature infants are admitted.

This study was observational single-center study; consist of a heterogeneous group of relatively stable premature infants born at different gestational ages, but appropriate PMA for oral feeding initiation and maintenance. The primary outcome of the study (Days from NG removal to target full oral feeds, median days of 2.0 vs. $2.5, p=0.13$, mean days $2.4 \pm 1.9$ vs. $4.4 \pm 5.8, p=0.051$ ) showed a trend for lower number of days in the early group, compared to the late group. Assuming parametric distribution of the variable, the difference between the groups has a small to medium effect size of 0.46 . With this effect size, a statistically significant difference in days from NG removal to target full oral feeds between the two groups can be obtained from a sample size of 148 (74 in each group) with $80 \%$ power $(\alpha=0.05, \beta=0.20)$. The decision to remove NG tube was not based on a standardized unit practice, but was based on the individual provider discretion, which can be a limitation in that it adds to the subjectivity in approaches. Variability in individual views among a large number of NICU providers potentially may have influenced the study group determination and the outcomes observed. Generalization of these findings to premature infants with significant comorbidities or anomalies must be applied with caution, as our study participants did not include such patients.

In summary, lack of consensus regarding the timing of NG tube removal in relation to oral feeding competency in premature infants can be associated with practice variation among individual providers. Our study suggests that discontinuing NG tube when the oral feeding competency reaches $\sim 75 \%$ of prescribed enteral feeding volume in the preceding two days is safe and appropriate in stable low-risk premature infants. Keeping NG tube longer is not associated with any additional clinical benefits. As there is no harm in discontinuing NG tube at $\sim 75 \%$ oral feeding competency, as evidenced by similarities in growth and LOHS, at least in monitored clinical settings, this practice can be safe and may have potential benefits in lowering resource utilization, potentially reduce the length of hospitalization, increase opportunities for parent participation, all resulting in cutting down the healthcare costs.

\section{Compliance with ethical standards}

Conflict of interest The authors declare that they have no conflict of interest.

Publisher's note: Springer Nature remains neutral with regard to jurisdictional claims in published maps and institutional affiliations.

\section{References}

1. Newborn AAoPCoFa. Hospital discharge of the high-risk neonate. Pediatrics. 2008;122:1119-26.

2. Jadcherla SR, Wang M, Vijayapal AS, Leuthner SR. Impact of prematurity and co-morbidities on feeding milestones in neonates: a retrospective study. J Perinatol. 2010;30:201-8.

3. Pickler RH, Reyna BA, Wetzel PA, Lewis M. Effect of four approaches to oral feeding progression on clinical outcomes in preterm infants. Nurs Res Pract. 2015;2015:716828.

4. Walsh MC, Bell EF, Kandefer S, Saha S, Carlo WA, D'angio CT, et al. Neonatal outcomes of moderately preterm infants compared to extremely preterm infants. Pedia Res. 2017;82:297-304.

5. Lainwala S, Kosyakova N, Power K, Hussain N, Moore JE, Hagadorn JI, et al. Delayed achievement of oral feedings is associated with adverse neurodevelopmental outcomes at 18 to 26 months follow-up in preterm infants. Am J Perinatol. 2019. https://doi.org/10.1055/s-0039-1681059. [Epub ahead of print]

6. Lau C, Smith EO, Schanler RJ. Coordination of suck-swallow and swallow respiration in preterm infants. Acta Paediatr. 2003; 92:721-7.

7. Kirk AT, Alder SC, King JD. Cue-based oral feeding clinical pathway results in earlier attainment of full oral feeding in premature infants. J Perinatol. 2007;27:572-8.

8. Kamitsuka MD, Nervik PA, Nielsen SL, Clark RH. Incidence of nasogastric and gastrostomy tube at discharge is reduced after implementing an oral feeding protocol in premature $(<30$ weeks $)$ infants. Am J Perinatol. 2017;34:606-13.

9. Davidson E, Hinton D, Ryan-Wenger N, Jadcherla S. Quality improvement study of effectiveness of cue-based feeding in infants with bronchopulmonary dysplasia in the neonatal intensive care unit. J Obstet Gynecol Neonatal Nurs. 2013;42:629-40.

10. Greenspan JS, Wolfson MR, Holt WJ, Shaffer TH. Neonatal gastric intubation: differential respiratory effects between nasogastric and orogastric tubes. Pedia Pulmonol. 1990;8:254-8.

11. Bohnhorst B, Cech K, Peter C, Doerdelmann M. Oral versus nasal route for placing feeding tubes: no effect on hypoxemia and bradycardia in infants with apnea of prematurity. Neonatology. 2010;98:143-9.

12. Shiao SY, Youngblut JM, Anderson GC, DiFiore JM, Martin RJ. Nasogastric tube placement: effects on breathing and sucking in very-low-birth-weight infants. Nurs Res. 1995;44:82-88.

13. Peter CS, Wiechers C, Bohnhorst B, Silny J, Poets CF. Influence of nasogastric tubes on gastroesophageal reflux in preterm infants: a multiple intraluminal impedance study. J Pediatr. 2002;141:277-9.

14. Murthy SV, Funderburk A, Abraham S, Epstein M, DiPalma J, Aghai ZH. Nasogastric feeding tubes may not contribute to gastroesophageal reflux in preterm infants. Am J Perinatol. 2018;35:643-7.

15. Rogers SP, Hicks PD, Hamzo M, Veit LE, Abrams SA. Continuous feedings of fortified human milk lead to nutrient losses of fat, calcium and phosphorous. Nutrients. 2010;2:230-40.

16. Petersen SM, Greisen G, Krogfelt KA. Nasogastric feeding tubes from a neonatal department yield high concentrations of potentially pathogenic bacteria- even $1 \mathrm{~d}$ after insertion. Pediatr Res. 2016;80:395-400. 
17. Jadcherla SR, Dail J, Malkar MB, McClead R, Kelleher K, Nelin L. Impact of process optimization and quality improvement measures on neonatal feeding outcomes at an all-referral neonatal intensive care unit. JPEN J Parent Enter Nutr. 2016;40:646-55.

18. Lau C. Development of infant oral feeding skills: what do we know? Am J Clin Nutr. 2016;103:616S-621S.
19. Latal-Hajnal B, von Siebenthal K, Kovari H, Bucher HU, Largo RH. Postnatal growth in VLBW infants: significant association with neurodevelopmental outcome. J Pediatr. 2003;143:163-70.

20. Fucile S, Phillips S, Bishop K, Jackson M, Yuzdepski T, Dow K. Identification of a pivotal period in the oral feeding progression of preterm infants. Am J Perinatol. 2019;36:530-6. 